\title{
Infection Prevention in Pediatric Oncology and Hematopoietic Stem Cell Transplant Recipients
}

\author{
Ankhi Dutta and Ricardo Flores
}

Children with malignancies or those who are hematopoietic stem cell transplant (HSCT) recipients are at increased risk for healthcare-associated infections (HAI) due to their underlying diagnoses as well as the receipt of immunosuppressive medications that are part of their treatment. In addition, frequent visits to healthcare settings increase the risk for exposure to resistant organisms. Thus, while adherence to infection control and prevention measures recommended for all hospitalized children is essential, additional interventions to reduce the inherent risk of morbidity associated with HAIs are warranted in this special population.

\section{Infection Prevention in Pediatric Oncology Patients and Hematopoietic Stem Cell Transplant Recipients}

There are various factors which contribute to the increased susceptibility to infections in pediatric hematology/oncology (PHO) and HSCT patients, most prominent of them being disruption of cutaneous and mucosal barriers (oral, gastrointestinal, etc.), microbial gastrointestinal translocation, defects in cell-mediated immunity, and insufficient quantities and inadequate function of phagocytes. Goals of infection control and prevention in this population are based on mitigating the risk inherent with the underlying malignancy and associated treatments (i.e., chemotherapy,

\footnotetext{
A. Dutta $(\square)$

Department of Pediatrics, Section of Pediatric Infectious Diseases, Texas Children's Hospital and Baylor College of Medicine, Houston, TX, USA

e-mail: adutta@bcm.edu

R. Flores

Department of Pediatrics, Texas Children's Cancer and Hematology Centers and Baylor

College of Medicine, Houston, TX, USA

e-mail: rjflores@txch.edu
} 
radiation). This chapter discusses infection control and prevention measures specifically in patients with hematological malignancies as well as HSCT recipients.

\section{General Measures}

Hand hygiene and standard precautions during the care of PHO and HSCT patients are key components in reducing the risk of infections. Additional isolation precautions may also be undertaken depending on the pathogen isolated and/or symptoms that the patient is experiencing (e.g., contact precautions would be appropriate in patients experiencing diarrhea). Further information on general infection prevention measures can be found in Chap. 1 .

\section{Skin Care and Hygiene}

Minimizing injury to mucosal surfaces and decreasing heavy colonization of the skin reduce the likelihood of microbial invasion through these sites. Thus, the importance of meticulous skin care and daily inspection in PHO and HSCT patients is paramount and provides opportunities to identify areas of inflammation or breakdown early. Skin inspection should be done routinely, with special attention to highrisk areas like intravascular catheter insertion sites and the perineum. Rectal thermometers, digital rectal examinations, and suppositories should be avoided to prevent mucosal breakdown. As part of an effort to reduce colonization of cutaneous surfaces, daily chlorhexidine baths have been shown to reduce HAIs and transmission of multidrug-resistant organisms (MDRO) in oncology patients $[1,2]$. Chlorhexidine gluconate $(\mathrm{CHG})$ is a cationic bisbiguanide that serves as a topical antiseptic. CHG binds to negatively charged bacterial cell wall proteins altering the bacterial cell wall equilibrium and helps in reducing bacterial colonization of the skin [1]. Education of patients, families, and staff on the importance of these practices is key to compliance with this preventative strategy and should be made a priority.

\section{Oral Hygiene}

Many experts recommend a complete periodontal examination be performed prior to initiation of chemotherapy with reevaluations throughout the treatment course and after completion [3,4]. Oral mucositis, which can be considered an acute inflammation and/or ulceration of the oral/oropharyngeal mucus membranes, is a common adverse effect of chemotherapeutic agents. It can cause oral pain/discomfort as well as difficulties in eating, swallowing, and speech. Mucositis is most commonly caused by chemotherapeutic agents which prevent DNA synthesis such as methotrexate, 5-fluorouracil, and cytarabine, particularly in HSCT recipients. Oral rinses with normal saline or CHG-containing products are recommended 4-6 times 
per day to prevent oral mucositis [2, 3]. Patients with painful mucositis might not comply with oral care regimens, however, putting them at increased risk for infections from oral flora such as bacteremia due to viridans streptococci. Mouth rinses containing alcohol should be avoided because they can aggravate mucositis. Neutropenic patients should also be instructed to brush their teeth carefully in order to prevent gingival injury [3]. A regular soft toothbrush or an electric brush can be used to minimize trauma [3].

Any elective dental procedure should be ideally performed prior to starting chemotherapy and after discussion with the primary medical team. The absolute neutrophil count, platelet count, and stage of treatment should be considered before performing any dental procedures in this vulnerable population $[2,3]$.

\section{Central Line-Associated Bloodstream Infection (CLABSI) Prevention}

The presence of central venous catheters $(\mathrm{CVC})$ in this population puts them at risk for central line-associated bloodstream infection (CLABSI) and its related complications. CLABSI is the most commonly reported HAI in most pediatric series. Among all the pediatric HAI reported to National Healthcare Surveillance Network (NHSN), 15\% were from oncology units; Streptococcus viridans (15\%) and Klebsiella pneumoniaeloxytoca (12\%) were the two most common pathogens in this study [5]. In the NHSN report, antibiotic resistance was noted to be high in oncology units, including ampicillin and/or vancomycin resistance for Enterococcus faecium and fluoroquinolone resistance for Escherichia coli [5]. Although less than 4\% of Enterobacteriaceae were reported to have carbapenem resistance, the emergence of such organisms in this population is of significant concern [6]. Among Candida infections in this population, fluconazole resistance among non-C. albicans and non-C. parapsilosis isolates was up to $41 \%$, whereas fluconazole resistance in C. albicans and C. parapsilosis was <4\% [5].

Mucosal barrier injury (MBI)-associated laboratory-confirmed bloodstream infections (MBI-LCBI) have gained attention in recent years [7, 8]. These are CLABSIs related primarily to mucosal barrier injury (i.e., mucositis) and not due to the direct presence of the CVC per se. In the NHSN definition, a positive blood culture would qualify as a MBI-LCBI if it results from one or more groups of selected commensal organisms of the oral cavity or gastrointestinal tract and occurred in the presence of signs and symptoms consistent with mucosal barrier injury (MBI) in PHO or HSCT patients [7]. Eligible organisms for MBI-LCBI include Candida species, Enterococcus, Enterobacteriaceae, viridans group streptococci, other Streptococcus species, and anaerobes [7].

Specific guidelines for central line insertion and maintenance bundles have been proposed by the Centers for Disease Control and Prevention (CDC) and the Infectious Diseases Society of America (IDSA) to reduce the CLABSI rates and healthcare costs $[9,10]$. Several studies have demonstrated that a multifaceted approach reduces CLABSI rates in this population [11, 12] and includes 
standardizing CVC insertion practices and maintenance bundles, tracking CVC infections using standardized definitions, and using dedicated nursing staff or " $\mathrm{CVC}$ champions" specifically trained in CVC maintenance and tracking in conjunction with other infection control methods (including oral and hand hygiene, optimizing nurse/patient ratio, etc.). CLABSI is discussed in greater detail in Chap. 6.

\section{Environmental and Dietary Considerations}

The American Society for Blood and Marrow Transplantation recommends a low microbial diet for HSCT recipients [13]. There is little evidence, however, to suggest that this helps in PHO patients. Routine safety in handling and preparing food should be practiced by patients and parents. In general, eating unpasteurized milk/ cheese, undercooked meat, and raw fruits and vegetables is discouraged during periods of neutropenia to reduce incidence of infection. The need to minimize risk of infection, however, should be balanced with the nutritional needs and quality of life of the patient $[2,13]$.

\section{Pet Ownership}

Pets can be a great source of companionship and comfort to children; however, there are several diseases that can be transmitted by pets to these immunosuppressed hosts [14-16]. Certain animals like reptiles, birds, rodents, or other exotic animals that cannot be immunized and could carry unusual human pathogens should not be kept as pets in households with PHO or HSCT patients. Immunosuppressed patients should avoid petting zoos due to the risk of diseases secondary to enteric pathogens (such as Salmonella or Campylobacter) [13-16].

Dogs and cats, preferably more than 1 year old, are generally considered safe for PHO and HSCT patients. They should be routinely evaluated by veterinarians for diseases and their immunizations kept up-to-date. Extreme care should be taken to maintain hand hygiene during and after handling the pets [13-16]. Further information regarding pet therapy is available in Chap. 4.

\section{Prevention of Bacterial Infections: Antibiotic Prophylaxis in Neutropenic Patients Without Fever}

Studies performed in adult oncology patients have consistently shown the benefit of using prophylactic antibiotics in reducing the incidence of bacterial infections [17]. Levofloxacin prophylaxis in adults has been shown to reduce the incidence of fever, bacterial infection, hospitalization rates, and all-cause mortality [18, 19]. Based upon such data in adults, the IDSA Guidelines for the Use of Antimicrobial Agents in Neutropenic Patients with Cancer state that fluoroquinolone prophylaxis should be considered for high-risk patients with prolonged severe neutropenia [20]. 
Pediatric studies on antibiotic prophylaxis are limited. A pediatric pilot study on the use of ciprofloxacin prophylaxis for pediatric patients receiving delayed intensification therapy for acute lymphoblastic leukemia (ALL) showed a reduction in hospitalization, intensive care admission, and bacteremia when compared to controls [21]. In another study, levofloxacin prophylaxis in patients with ALL reduced the odds of febrile neutropenia, possible bacterial infection, and confirmed bloodstream infection by $\geq 70 \%$. It also reduced the use of other broad-spectrum antibiotics and the incidence of $C$. difficile infections [22]. In other studies, however, ciprofloxacin prophylaxis did not decrease the incidence of overall bacteremia or duration of fever or mortality in pediatric acute myelogenous leukemia (AML) patients [23]. Furthermore, increasing quinolone resistance among gram-negative organisms is a concern recently observed in the NHSN database of pediatric oncology patients with CLABSI [5]. In addition, the use of antimicrobial prophylaxis in PHO could increase the possibility of developing other MDROs, invasive fungal infections, or drug-related toxicities. Though some authors suggest that antibiotic prophylaxis should be considered in children undergoing induction chemotherapy for ALL, there is currently insufficient data to inform definitive guidelines for antibiotic prophylaxis to prevent bacterial infections in pediatric oncology patients [19-21]. Notably, an open-label randomized clinical trial was recently conducted of levofloxacin prophylaxis vs. no prophylaxis in children with AML, relapsed ALL, and HSCT recipients. Among patients with AML and relapsed ALL, prophylaxis was associated with a reduction in rates of bacteremia; there was a numeric reduction in bacteremia in the HSCT recipients, but this did not achieve statistical significance. It is unclear at this time how these new findings will influence practice and future guidelines [24].

\section{Prevention of Viral Infections}

Infections with common respiratory and gastrointestinal viruses can result in significant morbidity and mortality in PHO and HSCT patients. The most common respiratory viruses encountered include rhinovirus, coronavirus, adenovirus, RSV, parainfluenza, human metapneumovirus, and influenza. Common gastrointestinal viruses affecting both healthy and immunocompromised children include norovirus, rotavirus, enteric adenoviruses, and enteroviruses among others. Infection prevention strategies should include education provided to the patient and the family about hand hygiene, prevention techniques, avoidance of ill visitors, disease surveillance in the community and hospital, vaccination against influenza and prompt identification, and testing and treatment (if possible) of any respiratory viral illness. Implementation of routine infection control prevention policies on oncology wards should reduce transmission of common respiratory and gastrointestinal viruses. All visitors should be screened for any signs and symptoms of acute viral illness and restricted from visitation on the unit or contact with any immunocompromised hosts. Chapter 4 outlines infection control guidance for hospital visitors in greater detail. 


\section{Immunization of Healthcare Workers and Household Contacts}

Immunization of healthcare workers and household contacts needs special consideration in settings with $\mathrm{PHO}$ and HSCT patients. Given the immunosuppressed status of children with malignancy and/or HSCT, immunization of those closest to them at home and those caring for them in the hospital is critically important in preventing infections. Live attenuated vaccines contain a theoretical risk of being transmitted to an immunocompromised host. Live oral polio vaccine, which is no longer administered in the United States, is an absolute contraindication for people taking care of this high-risk population. However, data suggests that measles, mumps, and rubella (MMR), varicella zoster, and herpes zoster vaccines can be safely provided to healthcare workers and household contacts [25]. If healthcare personnel develop a rash that cannot be covered within the first 42 days following receipt of the varicella vaccine, they should avoid any contact with immunocompromised patients until all rash has crusted to avoid the potential risk of transmitting vaccine strain varicella to patients [25]. Infants living in households with persons who are immunocompromised including PHO and HSCT patients may be safely immunized against rotavirus; it is recommended, however, that immunocompromised persons avoid contact with the infant's diapers/stool for 4 weeks following vaccination to minimize risk of acquiring vaccine strain rotavirus infection [26]. An inactivated influenza vaccine is preferred for personnel taking care of immunocompromised children as opposed to live attenuated influenza vaccine [25]. Vaccination against other non-viral pathogens (such as pneumococcus or pertussis) by family members is another important method to minimize the risk of serious infection in PHO patients.

\section{Physical Measures to Prevent Fungal Infections}

Hospital environments are designed to minimize the potential for fungal disease in the highest-risk patients. High efficiency particulate air (HEPA) filters have been shown to reduce nosocomial infection in HSCT patients, and the CDC recommends HEPA filters in HSCT recipient's rooms. The rooms should also have directed airflow and positive air pressure and be properly ventilated ( $\geq 12$ air changes per hour) [2]. Avoidance of carpets and upholstery is also recommended. Since outbreaks secondary to Aspergillus have been reported during hospital renovation or construction, appropriate containment should be in place, and strict precautions should be taken to prevent exposure to patients during such periods [2]. Infection control and prevention departments should be involved in risk assessment, planning, and approval of all construction or renovation projects in healthcare facilities including inpatient units, clinics, and infusion centers caring for these patients [27].

\section{Fever and Neutropenia}

Cytotoxic chemotherapies and radiation therapy used in the treatment of malignancies are myelosuppressive and result in variable duration and severity of neutropenia. In addition, certain malignancies that originate from bone marrow precursors 
(i.e., leukemia) or metastasize to the bone marrow (e.g., lymphoma, neuroblastoma, and sarcomas) can result in a decreased number of normal blood cell precursors and consequent neutropenia. Hence, pediatric cancer and HSCT patients are frequently immunosuppressed and at risk for a wide range of pathogens.

Febrile neutropenia is a common condition in the $\mathrm{PHO} / \mathrm{HSCT}$ population. With regard to this entity, fever is defined as a single temperature $>38.3^{\circ} \mathrm{C}\left(101^{\circ} \mathrm{F}\right)$ or a temperature $\geq 38.0^{\circ} \mathrm{C}\left(100.4{ }^{\circ} \mathrm{F}\right)$ on two occasions 1 hour apart. Neutropenia is classified as mild (absolute neutrophil count $\left[\right.$ ANC] $>500-1000 / \mathrm{mm}^{3}$ ), moderate $\left(\right.$ ANC $\left.\geq 200-500 / \mathrm{mm}^{3}\right)$, or severe $\left(\right.$ ANC $<200 / \mathrm{mm}^{3}$ ). Febrile neutropenia (also known as fever and neutropenia) is the combination of these two events in the patient with malignancy or HSCT and is a common complication of cancer treatment. It has been estimated that $10-50 \%$ of patients with solid tumors and up to $80 \%$ of patients with hematologic malignancies will develop fever during at least one chemotherapy cycle associated with neutropenia [28]. Moreover, fever may be the only indication of a severe underlying infection as other signs and symptoms are often absent or minimized due to an inadequate inflammatory response. Therefore, physicians must be particularly aware of the infection risks, diagnostic methods, and antimicrobial therapies required for the management of febrile neutropenia in cancer patients.

In the majority of febrile episodes, a pathogen is not identified, with a clinically documented infection occurring in only $20-47 \%$ of cases. Of these patients, bacteremia occurs in 10-25\%, with most episodes seen in the setting of prolonged and/or profound neutropenia (ANC $<100$ neutrophils $/ \mathrm{mm}^{3}$ ) $[29,30]$. On the other hand, the most common sites of focal infection include the gastrointestinal tract, lung, and skin [31].

\section{Common Organisms}

Over the past five decades, the rates, antibiotic resistance, and epidemiologic spectrum of bloodstream pathogens isolated from febrile neutropenic patients have changed substantially under the selective pressure of broad-spectrum antimicrobial therapy and/or prophylaxis $[32,33]$. Early in the development of cytotoxic chemotherapies, during the 1960s and 1970s, gram-negative pathogens predominated in febrile neutropenia. Subsequently, during the 1980s and 1990s, gram-positive organisms became more common as use of indwelling plastic venous catheters became more prevalent, which can allow for colonization and subsequent infection by gram-positive skin flora [31,34]. Gram-positive bacteria currently account for $60-70 \%$ of culture-positive infections in pediatric cancer patients [5].

Importantly, a recent systematic review of the epidemiology and antibiotic resistance of pathogens causing bacteremia in cancer patients since 2008 showed a recent shift from gram-positive to gram-negative organisms [35]. The main causes for this new trend are to be determined, but the use and duration of antibiotic prophylaxis are an important factor to consider as the incidence of gram-negative bacteria was significantly higher in groups who did not receive antibiotic prophylaxis. The use of antibiotic prophylaxis, however, may conceivably select for resistant organisms; increasing rates of antibiotic resistance in both gram-negative and 
Table 16.1 Common bacterial pathogens in neutropenic patients

Gram-positive bacteria

Staphylococci (coagulase-negative staphylococci and Staphylococcus aureus)

Enterococci (E. faecium and E. faecalis)

Streptococci (viridans group streptococci, S. pneumoniae, and S. pyogenes)

Bacillus species

Gram-negative bacteria

Enterobacteriaceae (Escherichia coli, Enterobacter species, Klebsiella species, Citrobacter

species, etc.)

Non-fermenting gram-negative rods (Pseudomonas aeruginosa and Stenotrophomonas

species)

Acinetobacter species

Modified from [20, 29, 30]

gram-positive bacteria have been reported in the global community as well as the cancer population and are of significant concern $[5,31,35]$.

Overall, the most common blood isolate in the setting of febrile neutropenia is coagulase-negative staphylococci. Other less common blood isolates include Enterobacteriaceae, non-fermenting gram-negative bacteria (such as Pseudomonas), S. aureus, and streptococci (see Table 16.1). Providers should review the local data at their institution for prevalent blood isolates and antimicrobial susceptibility profiles.

\section{Stratification and Management of Neutropenic Patients}

Management of febrile neutropenia continues to evolve given the awareness that interventions previously considered standard of care (such as inpatient treatment with intravenous broad-spectrum antibiotics) may not be necessary nor appropriate for all patients [36]. It has become increasingly important to identify patients at high risk of infectious complications requiring more aggressive management and monitoring (i.e., inpatient setting with intravenous antibiotics). In addition, clinicians may be able to identify low-risk patient populations who may be managed in a less aggressive and more cost-effective manner (i.e., outpatient setting and/or with oral antibiotics). In order to address these issues, algorithmic approaches to neutropenic fever, infection prophylaxis, diagnosis, and treatment have been developed [20, 37-39].

It is well established that stratification of patients to determine the risk for complications of severe infection should be undertaken at presentation of fever [20,37]. This determines the type of empiric antibiotic therapy (oral vs. intravenous), venue of treatment (inpatient vs. outpatient), and duration of antibiotic therapy.

Generally, the risk for serious infection is directly related to the degree and duration of neutropenia. Pediatric patients with mild (ANC $\geq 500)$ and brief periods of neutropenia ( $<7$ days) are less likely to have infectious complications than those 
with moderate to severe neutropenia (ANC $\leq 500)$ lasting more than 7 days. Similarly, the risk for bacteremia and septicemia increases dramatically when the ANC is $<200$. Infectious complications that are more common with severe and prolonged neutropenia include bacteremia, pneumonitis, cellulitis, and abscess formation.

\section{Risk Stratification}

It is important to consider individual patient risk incorporating the latest recommendations for the management of neutropenic fever in children with cancer and HSCT $[37,38]$. Patients are generally stratified as either high or low risk as follows:

1. High-risk patients - anticipated prolonged ( $>7$ days duration) and profound neutropenia (ANC $<100$ cells $/ \mathrm{mm}^{3}$ following cytotoxic chemotherapy) and/or significant medical comorbid conditions, including hypotension, pneumonia, new-onset abdominal pain, or neurologic changes [20]

2. Low-risk patients - anticipated brief ( $<7$ days duration) neutropenic periods in those with no or few comorbidities [20]

In addition, risk classification may be based on the Multinational Association for Supportive Care in Cancer (MASCC) score (Table 16.2) [40]. A MASCC risk score of $\geq 21$ is recommended as the threshold for definition of low risk, with $6 \%$ of such patients developing serious medical complications compared to $39 \%$ of those scoring $<21$ [40]. However the MASCC score was developed and validated in adults and has not been validated in a pediatric population.

Table 16.2 MASCC

risk-index score

\begin{tabular}{l|c}
\hline Patient characteristic & Assigned weight \\
\hline Burden of illness: no or mild symptoms & 5 \\
\hline Absence of hypotension & 5 \\
\hline $\begin{array}{l}\text { No chronic obstructive pulmonary } \\
\text { disease }\end{array}$ & 4 \\
\hline $\begin{array}{l}\text { Solid tumor or no previous fungal } \\
\text { infection }\end{array}$ & 4 \\
\hline No dehydration & 3 \\
\hline Burden of illness: moderate symptoms & 3 \\
\hline Outpatient status & 3 \\
\hline Age $<60$ years & 2 \\
\hline
\end{tabular}

Legend: The MASCC index score is calculated as the sum of the above variables. For patients meeting any given criteria, they are assigned weighted points specific to that individual criteria

${ }^{a}$ Represent mutually exclusive categories 


\section{Evaluation}

Upon presentation of the neutropenic fever patient, blood cultures should be obtained from all lumens of central venous catheters and consideration given to concomitant cultures from peripheral blood. Urinalysis and urine culture should be considered in patients with a readily available midstream specimen. Chest radiography should only be obtained in patients with signs or symptoms of respiratory infection. Other diagnostic tests should be performed based upon the presenting signs and symptoms. In children with upper respiratory symptoms, several viruses should be considered in the differential diagnosis as discussed above with the inclusion of molecular diagnostic studies as appropriate. Patients with gastrointestinal symptoms might be evaluated for gastrointestinal pathogens including Clostridium diffcile, particularly if they have recently been treated with antibiotics (see Chap. 12).

\section{Treatment}

The consensus in the field is for all patients considered to be at high risk by MASCC or by clinical criteria to be treated as inpatients with empiric IV antibiotic therapy. Carefully selected low-risk patients may be candidates for oral and/or outpatient empiric antibiotic therapy. Table 16.3 summarizes the recommendation for the management of febrile neutropenia based on recommendations of the IDSA and the International Pediatric Fever and Neutropenia Guideline Panel. Importantly, in neutropenic febrile patients with an obvious source of infection on clinical exam, management should be tailored to that source.

Of note, adequate antibiotic stewardship is of utmost importance during the treatment of neutropenic patients in order to decrease the incidence of antibiotic-related

Table 16.3 Summary of recommendations for management of fever and neutropenia (FN) in pediatric hematology/oncology and HSCT patients

\begin{tabular}{l|l}
\hline $\begin{array}{l}\text { Recommendation } \\
\text { Initial management }\end{array}$ & $\begin{array}{l}\text { Strength of recommendation } \\
\text { Quality of evidence }\end{array}$ \\
\hline $\begin{array}{l}\text { Risk stratification } \\
\text { Adopt a validated risk stratification strategy and incorporate } \\
\text { it into routine clinical management }\end{array}$ & $\begin{array}{l}\text { Strong recommendation } \\
\text { Low-quality evidence }\end{array}$ \\
\hline $\begin{array}{l}\text { Evaluation } \\
\text { Obtain blood cultures at the onset of FN from all lumens of } \\
\text { central venous catheters }\end{array}$ & $\begin{array}{l}\text { Strong recommendation } \\
\text { Low-quality evidence }\end{array}$ \\
\hline $\begin{array}{l}\text { Consider obtaining peripheral blood cultures concurrent } \\
\text { with central venous catheter cultures }\end{array}$ & $\begin{array}{l}\text { Weak recommendation } \\
\text { Moderate-quality evidence }\end{array}$ \\
\hline $\begin{array}{l}\text { Consider urinalysis and urine culture in patients in whom a } \\
\text { clean-catch, midstream specimen is readily available }\end{array}$ & $\begin{array}{l}\text { Weak recommendation } \\
\text { Low-quality evidence }\end{array}$ \\
\hline $\begin{array}{l}\text { Obtain chest radiography only in patients with respiratory } \\
\text { signs or symptoms }\end{array}$ & $\begin{array}{l}\text { Strong recommendation } \\
\text { Moderate-quality evidence }\end{array}$ \\
\hline
\end{tabular}


Table 16.3 (continued)

\begin{tabular}{l|l}
\hline $\begin{array}{l}\text { Recommendation } \\
\text { Treatment }\end{array}$ & $\begin{array}{l}\text { Strength of recommendation } \\
\text { Quality of evidence }\end{array}$ \\
\hline High-risk patients & \\
\hline $\begin{array}{l}\text { Use monotherapy with an antipseudomonal } \beta \text {-lactam, a } \\
\text { fourth generation cephalosporin, or a carbapenem as } \\
\text { empirical therapy in pediatric high-risk FN depending on the } \\
\text { local prevalence of multidrug-resistant gram-negative rods }\end{array}$ & $\begin{array}{l}\text { Strong recommendation } \\
\text { High-quality evidence }\end{array}$ \\
\hline $\begin{array}{l}\text { Reserve addition of a second gram-negative agent or a } \\
\text { glycopeptide for patients who are clinically unstable, when a } \\
\text { resistant infection is suspected, or for centers with a high } \\
\text { rate of resistant pathogens }\end{array}$ & $\begin{array}{l}\text { Strong recommendation } \\
\text { Moderate-quality evidence }\end{array}$ \\
\hline $\begin{array}{l}\text { Low-risk patients } \\
\begin{array}{l}\text { Consider initial or step-down outpatient management if the } \\
\text { infrastructure is in place to ensure careful monitoring and } \\
\text { follow-up }\end{array}\end{array}$ & $\begin{array}{l}\text { Weak recommendation } \\
\text { Moderate-quality evidence }\end{array}$ \\
\hline $\begin{array}{l}\text { Consider oral antibiotic administration if the child is able to } \\
\text { tolerate this route of administration reliably }\end{array}$ & $\begin{array}{l}\text { Weak recommendation } \\
\text { Moderate-quality evidence }\end{array}$ \\
\hline
\end{tabular}

Adapted from $[20,37,41]$

adverse drug events, prevalence of antibiotic resistance, and decrease treatment costs. Blood cultures must be closely monitored, and once a microorganism has been identified, an appropriate plan for antibiotic de-escalation and/or treatment duration should be promptly instituted.

\section{Invasive Fungal Infections}

Invasive fungal diseases (IFD) are one of the leading causes of morbidity and mortality in PHO and HSCT patients and present many diagnostic and therapeutic challenges.

\section{Risk Factors}

One of the principal risk factors contributing to the development of IFD relates to the patient's oncologic diagnosis. Patients with AML and high-risk and relapsed ALL, recipients of allogenic HSCT, and those with chronic or severe acute graft-versushost disease (GVHD) are at the highest risk of IFD [42, 43]. Often a combination of other risk factors is present in these patients which may include prolonged neutropenia, high-dose corticosteroid use, immunosuppressive therapy, parenteral nutrition, presence of a CVC, preceding antibiotic therapy, presence of bacterial coinfection, oral mucositis, and admission to an intensive care unit [44, 45]. The highest risk of IFD is during periods of profound neutropenia which for HSCT recipients occurs during the first 30 days posttransplant and during neutrophil engraftment [46]; for PHO patients, the highest risk period is during induction chemotherapy [46]. 
In an era of growing prophylactic antifungal use, children receiving mold-active agents have been shown to be at higher risk of non-Aspergillus species fungal infection [43]. Voriconazole prophylaxis in adults has been shown to be an independent risk factor for mucormycoses [47]. Likewise, breakthrough trichosporonosis has also been reported in patients receiving micafungin as prophylaxis [48]. These phenomena are likely in part related to the selection of fungi with reduced intrinsic susceptibility to the prophylactic agent.

\section{Species Distribution}

The most common IFD are invasive aspergillosis (IA) and invasive candidiasis (IC), with a recent upward trend seen in non-Aspergillus mold infections [43-45]. Among Aspergillus species, A. fumigatus is the most common, followed by A. flavus and A.niger [45]. Among non-Aspergillus molds, mucormycoses (Rhizopus, Mucor, Absidia) are most frequently reported followed by a number of other species (e.g., Fusarium, Scedosporium, Curvularia, Exserohilum, etc.) [45].

Among IC, C. albicans is the single most common Candida species, but nonalbicans Candida species (especially $C$. parapsilosis and $C$. tropicalis) have been increasingly reported among this population [49].

\section{Clinical Presentation}

IFD should be suspected in patients with fever and neutropenia lasting for more than 4 days without any identifiable cause [20]. IC can present as septic shock or may have more non-specific findings such as fever, cough, nausea/vomiting, abdominal pain, and cutaneous lesions depending on the site of involvement. In children, the most common sites of IC are the lungs, liver, and spleen, but dissemination can occur to the other organs including the heart, eyes, or brain. Disseminated disease is an independent risk factor for death in children with IC [50].

The primary sites of IA are the lungs, skin, and sinuses [45]. The clinical presentation of fungal rhinosinusitis may include fever, rhinorrhea, nasal congestion, and facial pain; many cases, however, may not present with any symptoms and may be diagnosed based on imaging performed in a persistently febrile patient with profound and prolonged neutropenia. Cutaneous lesions can present as macules, papules, or nodular ulcerative lesions with or without surrounding erythema and tenderness.

Clinical presentation secondary to other molds, such as Fusarium or Scedosporium, is indistinguishable from IA. Mucormycoses deserve special mention since dissemination and death are higher due to IFD caused by these species when compared to IA [51].

\section{Diagnosis}

Early recognition and prompt treatment of IFD are crucial for optimal management. Diagnostic tests should include blood cultures (though often with low sensitivity), 
cultures of appropriate sterile sites (such as urine or CSF), and diagnostic biopsies of involved sites for culture and histopathology. Fungal biomarkers can be used as both a screening test during high-risk periods and adjunct diagnostic test in patients with suspected IFD, especially during the periods of prolonged fever and neutropenia. Galactomannan (GM) is a cell wall component released by Aspergillus species which can be detected in blood, bronchoalveolar lavage fluid, and cerebrospinal fluid. A cutoff value of a GM optical index of $\geq 0.5$ in blood and a bronchoalveolar lavage fluid level of $\geq 1$ is considered a positive test, though an optimum cutoff value is not well defined in children [52,53]. Invasive fungal disease due to fungi other than Aspergillus species may have negative galactomannan tests. $\beta$-D-Glucan is a cell wall component found in many (but not all) species of fungi, and an elevated serum $\beta$-D-glucan assay can be caused by IC, IA, and other molds [53, 54]. The optimum cutoff value of $\beta$-Dglucan for a positive test is unknown in children, but $\geq 80 \mathrm{pg} / \mathrm{ml}$ is used in most studies [54]. Both GM and $\beta$-D-glucan assays have variable sensitivity and specificity among children and should be interpreted with caution. The sensitivity of GM has been reported to range from 65 to $82 \%$ in children with malignancy and IA [55, 56]; by contrast the $\beta$-D-glucan assay has high sensitivity for IFD ( $90 \%)$ but suffers from poor specificity [57]. False-positive $\beta$-D-glucan can be due to systemic bacterial or viral coinfection, receipt of antibiotics (such as piperacillin-tazobactam or amoxicillinclavulanate), hemodialysis, receipt of albumin or intravenous immunoglobulin, material containing glucan, oral mucositis, and other GI mucosal breakdowns [54]. Other PCR-based fungal diagnostic tests are under investigation but have low sensitivity and specificity.

GM and $\beta$-D-glucan monitoring twice weekly is suggested to evaluate treatment response in those with confirmed/probable disease and as a screening tool in patients at high risk for IFD [52, 53]. All PHO and HSCT patients with febrile neutropenia that persists beyond 4 days and/or those with suspected IFD should undergo computed tomography of the chest, abdomen, and pelvis and of other areas if indicated [53]. The most common findings on imaging suggestive of IFD are pulmonary nodules, especially those with a halo sign, air crescent sign, or cavitations. Hepatosplenic and renal nodules should also raise suspicion of IFD. Other studies to consider include an echocardiogram and dilated retinal examination, especially in patients with disseminated candidiasis. If symptoms of sinusitis or new lesions on the palate are present, a prompt nasal endoscopic examination and CT of sinuses are warranted.

\section{Antifungal Prophylaxis}

There are three main classes of antifungals used in patients with IFD: (1) polyenes, which include amphotericin B (AmB) and its lipid formulations (liposomal AmB is most commonly used in PHO and HSCT patients); (2) triazoles (fluconazole, itraconazole, voriconazole, and posaconazole); and (3) echinocandins (caspofungin, micafungin, anidulafungin). Antifungal prophylaxis should be considered in patients who are at high risk for IFD including HSCT recipients and those undergoing intensive remission-induction therapy or salvage-induction therapy [46, 53]. A high incidence of IFD has been reported in children with AML (newly diagnosed and 
relapsed) [58] and patients with relapsed ALL [46], and such patients may be considered candidates for prophylaxis. Among HSCT recipients, those with an unrelated donor or a partially matched donor are at higher risk of IFD [46].

Recent studies show that children with AML receiving antifungal prophylaxis have reduced rates of induction mortality and resource utilization compared to those who did not receive prophylaxis [59]. Posaconazole was found to be superior to fluconazole or itraconazole in reducing incidence of IFD in children [60]. Echinocandins have been shown to be as or more effective for IFD prophylaxis than triazoles, especially in HSCT recipients, with less adverse effects and can be an alternative option for prophylaxis [46].

The IDSA and the European Conference on Infections in Leukemia (ECIL-4) recommend using posaconazole, voriconazole, or micafungin during prolonged neutropenia to prevent IFD [20,53]. Posaconazole is recommended for prophylaxis in patients with GVHD who are at high risk of IA [53]. Variable absorption of oral azoles in children should be taken into consideration when choosing oral antifungals.

\section{Treatment}

For patients with prolonged fever and neutropenia without an alternative explanation, consideration must be given to the possibility of an active fungal infection. Empiric antifungal therapy should be considered for neutropenic patients with persistent or recurrent fevers after 4-7 days of antibiotic therapy and whose overall duration of neutropenia is expected to be $>7$ days [20]. In low-risk patients, routine use of empiric antifungals is not recommended [20]. Liposomal amphotericin B or an echinocandin, both of which are fungicidal, are the first-line therapy for empiric antifungal treatment [20]. There is insufficient data to provide specific guidance for patients with concern for a new fungal infection who are already receiving moldactive (i.e., anti-Aspergillus) prophylaxis; however, some experts suggest switching to a different mold-active antifungal [18].

Surgical debridement of any fungal lesions or abscesses and prompt removal of $\mathrm{CVC}$ in the event of fungemia are crucial to reduce the progression of IFD.

Therapeutic drug monitoring (TDM) should be performed for patients receiving voriconazole, itraconazole, and posaconazole. There is extreme variability in triazole serum levels among pediatric patients owing to diversity in bioavailability in this population. For voriconazole TDM, a serum trough level between 1 and 5 $\mathrm{mcg} / \mathrm{dl}$ has been considered safe and effective in preventing breakthrough IFD in children [53]. For posaconazole, a trough level of $0.7 \mathrm{mg} / \mathrm{L}-1 \mathrm{mg} / \mathrm{L}$ has been shown to be effective [53]. Due to increased toxicity associated with vinca alkaloids, high doses of cyclophosphamide, and anthracyclines, azoles should not be co-administered with these agents.

The antifungal agents most commonly used in children with PHO and HSCT and their indications are noted below (Table 16.4). Although combination antifungals are not well studied in children, they are used frequently in this population. Pediatric 


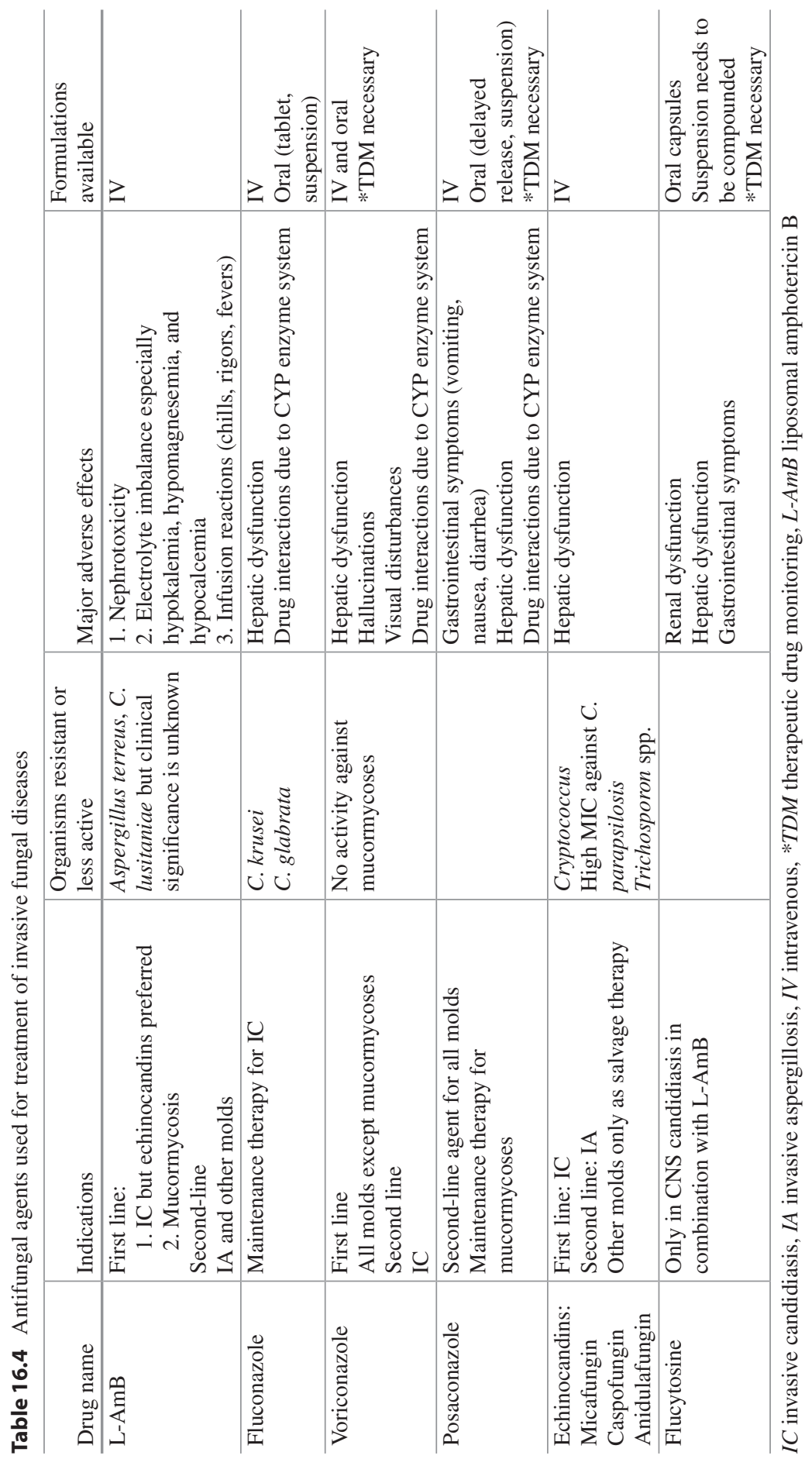


data are variable regarding the benefit of combination antifungal therapy but overall report an increase in adverse events [45]; the risk of systemic toxicity must therefore be taken into account when considering the use of antifungal combinations. Combination therapy could be considered in patients with refractory disease or as salvage therapy. Granulocyte transfusions for profound or persistent neutropenia, adjunctive cytokines (e.g., granulocyte colony-stimulating factor [GCSF]), and reduction of immunosuppression and tapering of steroids are recommended as an adjunct to antifungal agents in the treatment of IFD [20].

In summary, children and adolescents with malignancy have additional risk factors for healthcare-associated infections. Meticulous attention to personal and oral hygiene, diet, environmental safety, and appropriate immunizations should be practiced in this high-risk population. The use of antimicrobial prophylaxis should be considered in periods of severe neutropenia to prevent bacterial and fungal infections as necessary. Prompt diagnosis and management strategies to prevent infectious complications are key to preventing morbidity and mortality in these immunocompromised hosts.

\section{References}

1. Raulji CM, et al. Daily bathing with chlorhexidine and its effects on nosocomial infection rates in pediatric oncology patients. Pediatr Hematol Oncol. 2015;32(5):315-21.

2. Thom KA, Kleinberg M, Roghmann MC. Infection prevention in the cancer center. Clin Infect Dis. 2013;57(4):579-85.

3. Padmini C, Bai KY. Oral and dental considerations in pediatric leukemic patient. ISRN Hematol. 2014;2014:895721.

4. American Academy of Pediatric Dentistry Clinical Affairs, C. and A. American Academy of Pediatric Dentistry Council on Clinical. Guideline on dental management of pediatric patients receiving chemotherapy, hematopoietic cell transplantation, and/or radiation. Pediatr Dent. 2005;27(7 Suppl):170-5.

5. Lake JG, et al. Pathogen distribution and antimicrobial resistance among pediatric healthcareassociated infections reported to the national healthcare safety network, 2011-2014. Infect Control Hosp Epidemiol. 2018;39(1):1-11.

6. Af Sandeberg M, et al. Antibiotic use during infectious episodes in the first 6 months of anticancer treatment-A Swedish cohort study of children aged 7-16 years. Pediatr Blood Cancer. 2017;64(7):e26397.

7. See I, et al. Mucosal barrier injury laboratory-confirmed bloodstream infection: results from a field test of a new National Healthcare Safety Network definition. Infect Control Hosp Epidemiol. 2013;34(8):769-76.

8. Torres D, et al. The Centers for Disease Control and Prevention definition of mucosal barrier injury-associated bloodstream infection improves accurate detection of preventable bacteremia rates at a pediatric cancer center in a low- to middle-income country. Am J Infect Control. 2016;44(4):432-7.

9. Marschall J, et al. Strategies to prevent central line-associated bloodstream infections in acute care hospitals: 2014 update. Infect Control Hosp Epidemiol. 2014;35(Suppl 2):S89-107.

10. O'Grady NP, et al. Guidelines for the prevention of intravascular catheter-related infections. Clin Infect Dis. 2011;52(9):e162-93.

11. Bundy DG, et al. Preventing CLABSIs among pediatric hematology/oncology inpatients: national collaborative results. Pediatrics. 2014;134(6):e1678-85. 
12. Dandoy CE, et al. Rapid cycle development of a multifactorial intervention achieved sustained reductions in central line-associated bloodstream infections in haematology oncology units at a children's hospital: a time series analysis. BMJ Qual Saf. 2016;25(8):633-43.

13. Tomblyn M, et al. Guidelines for preventing infectious complications among hematopoietic cell transplantation recipients: a global perspective. Biol Blood Marrow Transplant. 2009;15(10):1143-238.

14. Gurry GA, et al. High rates of potentially infectious exposures between immunocompromised patients and their companion animals: an unmet need for education. Intern Med J. 2017;47(3):333-5.

15. Hemsworth S, Pizer B. Pet ownership in immunocompromised children--a review of the literature and survey of existing guidelines. Eur J Oncol Nurs. 2006;10(2):117-27.

16. Steele RW. Should immunocompromised patients have pets? Ochsner J. 2008;8(3):134-9.

17. Hammond SP, Baden LR. Antibiotic prophylaxis for patients with acute leukemia. Leuk Lymphoma. 2008;49(2):183-93.

18. Bucaneve $\mathrm{G}$, et al. Levofloxacin to prevent bacterial infection in patients with cancer and neutropenia. N Engl J Med. 2005;353(10):977-87.

19. Cullen M, et al. Antibacterial prophylaxis after chemotherapy for solid tumors and lymphomas. N Engl J Med. 2005;353(10):988-98.

20. Freifeld AG, et al. Clinical practice guideline for the use of antimicrobial agents in neutropenic patients with cancer: 2010 update by the Infectious Diseases Society of America. Clin Infect Dis. 2011;52(4):427-31.

21. Yousef AA, et al. A pilot study of prophylactic ciprofloxacin during delayed intensification in children with acute lymphoblastic leukemia. Pediatr Blood Cancer. 2004;43(6):637-43.

22. Wolf J, et al. Levofloxacin prophylaxis during induction therapy for pediatric acute lymphoblastic leukemia. Clin Infect Dis. 2017;65(11):1790-8.

23. Felsenstein $\mathrm{S}$, et al. Clinical and microbiologic outcomes of quinolone prophylaxis in children with acute myeloid leukemia. Pediatr Infect Dis J. 2015;34(4):e78-84.

24. Alexander S, Fisher RT, Gaur AH, et al. Effect of levofloxacin prophylaxis on bacteremia in children with acute leukemia or undergoing hematopoietic stem cell transplantation: a randomized clincial trial. JAMA. 2018;320:995-1004.

25. Shefer A, Strikas R, Bridges CB. Updated recommendations of the Advisory Committee on Immunization Practices for healthcare personnel vaccination: a necessary foundation for the essential work that remains to build successful programs. Infect Control Hosp Epidemiol. 2012;33(1):71-4.

26. American Academy of Pediatrics. Rotavirus infections. In: Kimberlin DW, et al., editors. 2018 redbook: report of the committee on infectious diseases. Grove Village: American Academy of Pediatrics; 2018. p. 700-4.

27. Sehulster L, et al. Guidelines for environmental infection control in health-care facilities. Recommendations of $\mathrm{CDC}$ and the Healthcare Infection Control Practices Advisory Committee (HICPAC). MMWR Recomm Rep. 2003;52(RR-10):1-42.

28. Klastersky J. Management of fever in neutropenic patients with different risks of complications. Clin Infect Dis. 2004;39(Suppl 1):S32-7.

29. Petty LA, et al. Repeated blood cultures in pediatric febrile neutropenia: would following the guidelines alter the outcome? Pediatr Blood Cancer. 2016;63(7):1244-9.

30. Hakim $\mathrm{H}$, et al. Etiology and clinical course of febrile neutropenia in children with cancer. J Pediatr Hematol Oncol. 2009;31(9):623-9.

31. Ramphal R. Changes in the etiology of bacteremia in febrile neutropenic patients and the susceptibilities of the currently isolated pathogens. Clin Infect Dis. 2004;39(Suppl 1):S25-31.

32. Jones RN. Contemporary antimicrobial susceptibility patterns of bacterial pathogens commonly associated with febrile patients with neutropenia. Clin Infect Dis. 1999;29(3):495-502.

33. Irfan $\mathrm{S}$, et al. Emergence of Carbapenem resistant Gram negative and vancomycin resistant Gram positive organisms in bacteremic isolates of febrile neutropenic patients: a descriptive study. BMC Infect Dis. 2008;8:80. 
34. Zinner SH. Changing epidemiology of infections in patients with neutropenia and cancer: emphasis on gram-positive and resistant bacteria. Clin Infect Dis. 1999;29(3):490-4.

35. Montassier E, et al. Recent changes in bacteremia in patients with cancer: a systematic review of epidemiology and antibiotic resistance. Eur J Clin Microbiol Infect Dis. 2013;32(7):841-50.

36. Kumar P, et al. Management of febrile neutropenia in malignancy using the MASCC score and other factors: feasibility and safety in routine clinical practice. Indian J Cancer. 2014;51(4):491-5.

37. Lehrnbecher $\mathrm{T}$, et al. Guideline for the management of fever and neutropenia in children with cancer and hematopoietic stem-cell transplantation recipients: 2017 update. J Clin Oncol. 2017;35(18):2082-94.

38. Hughes WT, et al. 2002 guidelines for the use of antimicrobial agents in neutropenic patients with cancer. Clin Infect Dis. 2002;34(6):730-51.

39. Taplitz RA, Kennedy EB, Flowers CR. Outpatient management of fever and neutropenia in adults treated for malignancy: American Society of Clinical Oncology and Infectious Diseases Society of America clinical practice guideline update summary. J Oncol Pract. 2018;14(4):250-5.

40. Klastersky J, et al. The Multinational Association for Supportive Care in Cancer risk index: a multinational scoring system for identifying low-risk febrile neutropenic cancer patients. J Clin Oncol. 2000;18(16):3038-51.

41. Freifeld AG, et al. Clinical practice guideline for the use of antimicrobial agents in neutropenic patients with cancer: 2010 update by the infectious diseases society of america. Clin Infect Dis. 2011;52(4):e56-93.

42. Castagnola E, et al. Invasive mycoses in children receiving hemopoietic SCT. Bone Marrow Transplant. 2008;41(Suppl 2):S107-11.

43. Wattier RL, et al. A prospective, international cohort study of invasive mold infections in children. J Pediatr Infect Dis Soc. 2015;4(4):313-22.

44. Corzo-Leon DE, et al. Epidemiology and outcomes of invasive fungal infections in allogeneic haematopoietic stem cell transplant recipients in the era of antifungal prophylaxis: a singlecentre study with focus on emerging pathogens. Mycoses. 2015;58(6):325-36.

45. Georgiadou SP, et al. Invasive mold infections in pediatric cancer patients reflect heterogeneity in etiology, presentation, and outcome: a 10-year, single-institution, retrospective study. $\mathrm{J}$ Pediatr Infect Dis Soc. 2012;1(2):125-35.

46. Dvorak CC, et al. Antifungal prophylaxis in pediatric hematology/oncology: new choices \& new data. Pediatr Blood Cancer. 2012;59(1):21-6.

47. Marty FM, Cosimi LA, Baden LR. Breakthrough zygomycosis after voriconazole treatment in recipients of hematopoietic stem-cell transplants. N Engl J Med. 2004;350(9):950-2.

48. Foster CE, et al. Trichosporonosis in pediatric patients with a hematologic disorder. J Pediatr Infect Dis Soc. 2017. https://doi.org/10.1093/jpids/pix031.

49. Steinbach WJ, et al. Results from a prospective, international, epidemiologic study of invasive candidiasis in children and neonates. Pediatr Infect Dis J. 2012;31(12):1252-7.

50. Zaoutis TE, et al. Risk factors for mortality in children with candidemia. Pediatr Infect Dis J. 2005;24(8):736-9.

51. Pana ZD, et al. Invasive mucormycosis in children: an epidemiologic study in European and non-European countries based on two registries. BMC Infect Dis. 2016;16(1):667.

52. Patterson TF, et al. Practice guidelines for the diagnosis and management of Aspergillosis: 2016 update by the Infectious Diseases Society of America. Clin Infect Dis. 2016;63(4):e1-e60.

53. Groll AH, et al. Fourth European Conference on Infections in Leukaemia (ECIL-4): guidelines for diagnosis, prevention, and treatment of invasive fungal diseases in paediatric patients with cancer or allogeneic haemopoietic stem-cell transplantation. Lancet Oncol. 2014;15(8):e327-40.

54. Pappas PG, et al. Clinical practice guideline for the management of Candidiasis: 2016 update by the Infectious Diseases Society of America. Clin Infect Dis. 2016;62(4):e1-50.

55. Dinand V, et al. Threshold of galactomannan antigenemia positivity for early diagnosis of invasive aspergillosis in neutropenic children. J Microbiol Immunol Infect. 2016;49(1):66-73. 
56. Hayden R, et al. Galactomannan antigenemia in pediatric oncology patients with invasive aspergillosis. Pediatr Infect Dis J. 2008;27(9):815-9.

57. Koltze A, et al. Beta-D-glucan screening for detection of invasive fungal disease in children undergoing allogeneic hematopoietic stem cell transplantation. J Clin Microbiol. 2015;53(8):2605-10.

58. Science M, et al. Guideline for primary antifungal prophylaxis for pediatric patients with cancer or hematopoietic stem cell transplant recipients. Pediatr Blood Cancer. 2014;61(3):393-400.

59. Fisher BT, et al. Antifungal prophylaxis associated with decreased induction mortality rates and resources utilized in children with new-onset acute myeloid leukemia. Clin Infect Dis. 2014;58(4):502-8.

60. Doring M, et al. Antifungal prophylaxis with posaconazole vs. fluconazole or itraconazole in pediatric patients with neutropenia. Eur J Clin Microbiol Infect Dis. 2015;34(6):1189-200. 